Proceeding Paper

\title{
Influence of Different Copper Treatment on the Formation of Single-Layer Graphene by CVD Method ${ }^{\dagger}$
}

\author{
Ivan Kondrashov 1,*, Maxim Komlenok 1, Pavel Pivovarov 1, Sergei Savin 2, Elena Obraztsova 1,3 \\ and Maxim Rybin 1,3,4
}

Citation: Kondrashov, I.; Komlenok, M.; Pivovarov, P.; Savin, S.; Obraztsova, E.; Rybin, M. Influence of Different Copper Treatment on the Formation of Single-Layer Graphene by CVD Method. 2021, 4 , 14. https://doi.org/10.3390/IOCN 2020-07839

Academic Editors: Ana María Díez-Pascual, Antonio Di Bartolomeo and Guanying Chen

Published: 11 November 2020

Publisher's Note: MDPI stays neutral with regard to jurisdictional claims in published maps and institutional affiliations.

Copyright: (c) 2020 by the authors. Licensee MDPI, Basel, Switzerland. This article is an open access article distributed under the terms and conditions of the Creative Commons Attribution (CC BY) license (http://creativecommons.org/licenses/by/4.0/).
1 Prokhorov General Physics Institute of the Russian Academy of Sciences, 38 Vavilov st., 119991 Moscow, Russia; komlyonok@mail.ru (M.K.); p_pivovarov@hotmail.com (P.P.); elobr@mail.ru (E.O.); rybmaxim@gmail.com (M.R.)

2 MIREA-Russian Technological University, 78 Vernadsky Avenue, 119454 Moscow, Russia; savin@mirea.ru

3 Moscow Institute of Physics and Technology, State University, 1 "A" Kerchenskaya st., 117303 Moscow, Russia

4 LLC “RUSGRAPHENE” 8 Lesnoy, Protvino, 142281 Moscow, Russia

* Correspondence: navi.soul@gmail.com

+ Presented at the 2nd International Online-Conference on Nanomaterials, 15-30 November 2020; Available online: https://iocn2020.sciforum.net/.

\begin{abstract}
Chemical vapor deposition synthesis of graphene on copper foil from methane is the most promising technology for industrial production. However, an important problem of the formation of the second and subsequent graphene layers during synthesis arises due to the strong roughness of the initial copper foil. Here we demonstrate the various approaches to prepare a smooth copper surface before graphene synthesis to reduce the formation of multi-layer graphene islands. Six methods of surface processing of copper foils are studied, and the decrease of the roughness from 250 to as low as $80 \mathrm{~nm}$ is achieved. The correlation between roughness and the formation of multi-layer graphene is demonstrated. Under optimized conditions of surface treatment, the content of the multi-layer graphene islands drops from $9 \%$ to $2.1 \%$. The quality and the number of layers of synthesized graphene are analyzed by Raman spectroscopy, scanning electron microscopy, and measurements of charge mobility.
\end{abstract}

Keywords: CVD synthesis; graphene monolayer; surface treatment; electrochemical polishing

\section{Introduction}

Chemical vapor deposition (CVD) synthesis of graphene on metal substrates is the most promising method for growing large-area films of high-quality [1]. Copper foil is usually chosen as a catalytic substrate for graphene synthesis since the single layer is easily grown due to the limited solubility of carbon in copper [2]. The quality of graphene depends not only on precursors and CVD parameters (pressure, temperature) but also on the properties of the copper substrate such as thickness, surface roughness, polycrystallinity, grain size, and the presence of surface impurities [3]. If the copper surface is well developed and has a strong roughness, then the formation of a second graphene layer during synthesis becomes more likely than on a smooth surface.

Copper foil is usually produced by mechanical rolling resulting in scratches along the rolling direction. As reported, the density and uniformity of graphene domains correlate with the surface roughness of the copper substrate [4]. One of the most efficient ways to grow an ideal graphene monolayer is to use a smooth single-crystal copper [5]. However, this approach is technologically difficult to implement and scale, since it requires the purchase of expensive single-crystal copper or recrystallization of a copper film. A simpler and more affordable option to reduce the formation of the second graphene layer is 
to process the surface of copper before the synthesis to reduce surface roughness. Different pretreatment methods have been investigated such as physical polishing [6], chemical etching [7], annealing [8], resolidification [9], and electrochemical polishing (ECP) [10] to smooth the surface. Mechanical polishing is a good method to get a smooth surface, but a thick copper substrate and the use of a special grinding machine are needed. Chemical treatment is effective for cleaning the surface of copper from impurities, but it is necessary to adjust the concentration of strong etchants based on nitric acid and the exposure time to avoid complete etching of copper. The annealing (resolidification) is a relatively simple method for smoothing the surface of copper. It is based on a rearrangement of surface copper atoms, which leads to the release of internal stresses and an increase in crystal size. However, this approach requires a high temperature close to the melting point of copper $\left(1085^{\circ} \mathrm{C}\right)$ and a long time for processing. $\mathrm{ECP}$ is the most effective method of cleaning and smoothing the surface of copper foil, which takes a few minutes and requires only a phosphoric acid and a power supply not exceeding 10 Watts.

\section{Materials and Methods}

\subsection{Pretreatment of Copper Substrates}

Alfa Aesar copper foil $(25 \mu \mathrm{m}, 99.8 \%)$ was used for various processing methods before the graphene synthesis. The chemical etching of the sample in nitric acid was performed in solution with a concentration of $\mathrm{H}_{2} \mathrm{O}: \mathrm{HNO}_{3}=2: 1$ for $60 \mathrm{~s}$. ECP was made using copper foil as anode placed in parallel to bulk copper substrate cathode in phosphoric acid for $60 \mathrm{~s}$. The annealing of the copper sample was performed at $1000^{\circ} \mathrm{C}$ in a hydrogen atmosphere under $100 \mathrm{mbar}$ for $5 \mathrm{~h}$.

\subsection{Copper Film Deposition}

The copper film was deposited on a single-crystal sapphire substrate in a UNIVEX 300 vacuum chamber at a pressure of $10-5$ mbar by passing an electric current through a molybdenum boat with copper metal pieces. The molybdenum boat was heated up to $1700-2000{ }^{\circ} \mathrm{C}$ and the copper evaporated and deposited on the target substrate. The thickness of the copper film was $1000 \mathrm{~nm}$ and was determined by the mass of metal placed into the boat.

\subsection{Graphene Growth Procedure}

The synthesis of graphene on copper foil has been well studied and various scientific groups have presented numerous options for the synthesis of graphene on copper foil under various conditions. The review [11] presents the basic principles of the formation of graphene on copper. We used a standard tube furnace and the main feature of the synthesis was the absence of gas flow. The following optimal parameters of graphene growth were determined in this configuration: pressure 100 mbar, gas concentration of $\mathrm{H}_{2}: \mathrm{CH}_{4}=$ 100:1, temperature $1000^{\circ} \mathrm{C}$ for $20 \mathrm{~min}$. Before the graphene formation, the copper foil was annealed at $1000{ }^{\circ} \mathrm{C}$ for $30 \mathrm{~min}$ in a hydrogen atmosphere with $100 \mathrm{mbar}$ to remove natural oxide from copper foil.

\subsection{Transfer of the Grown Graphene}

After the growth, the graphene was transferred from the copper to a $\mathrm{SiO}_{2} / \mathrm{Si}$ substrate using standard "wet" transfer technology. First, polymethylmethacrylate (PMMA, 4\% in anisole) was applied to the copper foil by the spin-coating method at $3000 \mathrm{rpm}$ for $45 \mathrm{~s}$. Then, the copper was etched with ammonium persulfate $\left(\left(\mathrm{NH}_{4}\right)_{2} \mathrm{~S}_{2} \mathrm{O}_{8}\right)$. Finally, a PMMA/graphene film was washed with DI water and transferred onto the target substrate, and PMMA was removed with acetone. 


\subsection{Sample Characterization}

The quality of graphene films and the number of islands with two-layered graphene were investigated using optical microscopy and Raman scattering. The electrical characteristics of the graphene films were obtained using the four-probe method and Hall effect measurements. The surface morphology of the copper foil was examined by a white-light interference microscope (ZYGO NewView 5000) and a scanning electron microscope (SEM-TESCAN Mira 3). The amount of the second layer on the surface area was calculated using graphics software for image analysis of transferred graphene films obtained using an optical microscope. The second and subsequent graphene layers transferred from copper foil onto $285 \mathrm{~nm} \mathrm{SiO} / / \mathrm{Si}$ substrate had a higher contrast (darker areas) in optical images than the monolayer. This difference was enough to set the monolayer as the base contrast and calculate the number of all darker spots as a percentage of the entire surface area.

\section{Results and Discussion}

For the fabrication of a high quality and uniform graphene monolayer by CVD technology on copper foil from carbonaceous gas, it is necessary to use copper foil with the lowest value of roughness. In this work, six methods were used to form the surface with minimal roughness. The result of the analysis of surface morphology after different treatments is shown in Figure 1.

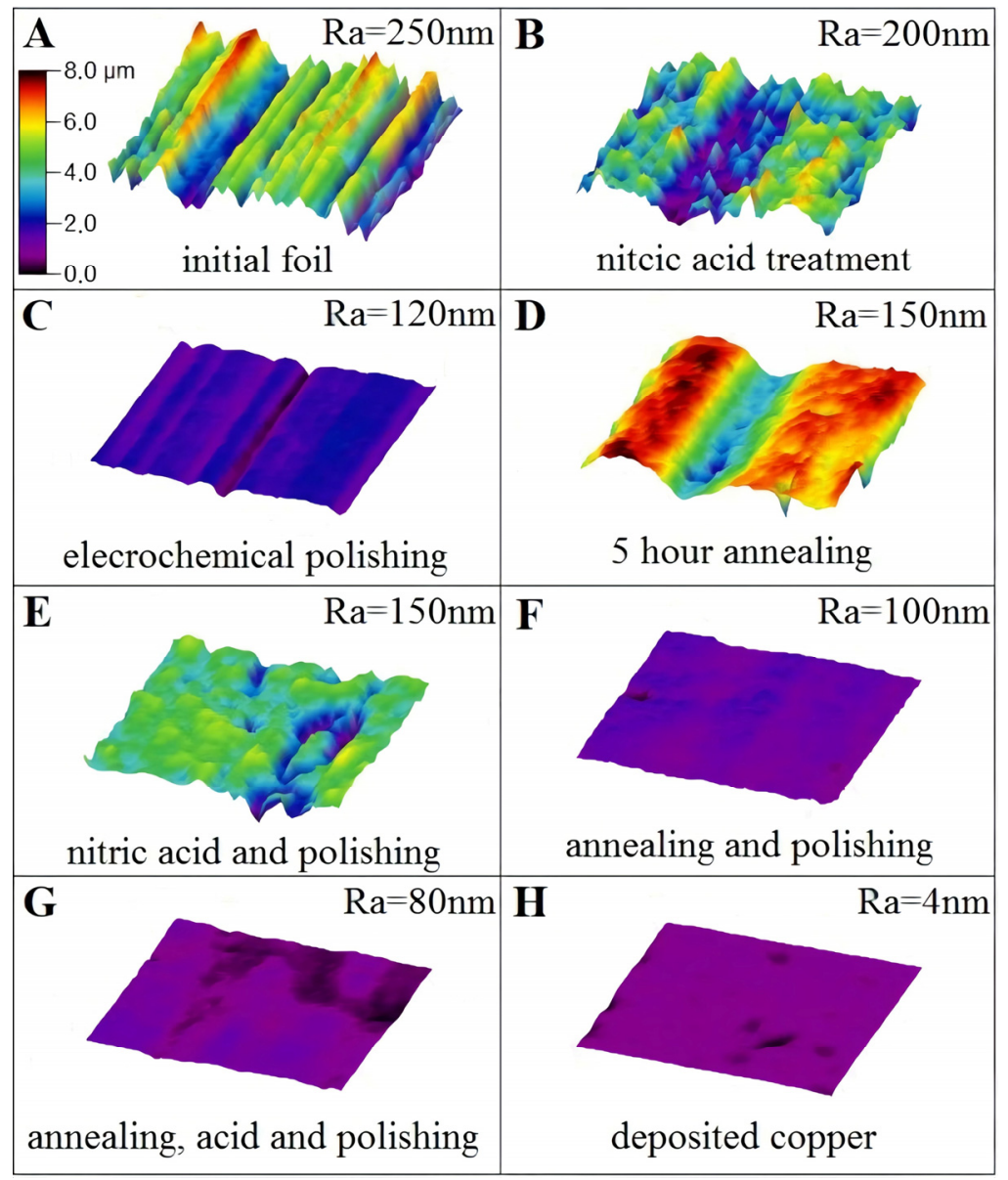

Figure 1. The roughness measurements of copper foil by interference microscope: (A)-without pre-treatment, (B) - nitric acid treatment, (C) - electrochemical polishing, (D)-hydrogen annealing for $5 \mathrm{~h},(\mathbf{E})$ - nitric acid treatment and electrochemical polishing, $(\mathbf{F})$-hydrogen annealing and electrochemical polishing, (G)-hydrogen annealing, nitric acid treatment and electrochemical polishing, $(\mathbf{H})$-deposited copper film. All measurements were done from $50 \times 50$ micrometers area. All figures have the same scale bar in color grade and it is indicated in (A). 
The initial copper foil has the arithmetic average roughness $R_{a}$ about $250 \mathrm{~nm}$ (Figure $1 \mathrm{~A}$ ). The treatment of sample in nitric acid leads to $R_{a}$ about $200 \mathrm{~nm}$ (Figure 1B), while ECP can provide $R_{a}=120 \mathrm{~nm}$ of the foil (Figure $1 \mathrm{C}$ ). The annealing process results in the same $\mathrm{R}_{\mathrm{a}}=$ $150 \mathrm{~nm}$ of copper foil as the combination of nitric acid treatment with further ECP, see Figure 1D,E, respectively. The combination of annealing and further polishing, as well as annealing and further acid treatment and ECP results in the roughness of 100 and $80 \mathrm{~nm}$ and is presented in Figure 1F,G, respectively. For comparison, the morphology of thin copper film ( $R_{a}=4 \mathrm{~nm}$ ) deposited on an optically flat single-crystal sapphire substrate is shown in Figure $1 \mathrm{H}$.

The process of nucleation of second and more layers of graphene and its correlation with defects (impurities) and the roughness of the copper surface is investigated using images obtained by electron microscope (Figure 2). The graphene film synthesized on initial copper foils without any processing has different impurities that look like black dots in the SEM image (Figure 2A). Almost $10 \%$ of the total area is occupied by islands of dark gray color, which correspond to double-layer graphene. The copper foil treated by a combination of annealing and ECP with synthesized graphene which has the lowest density of the few layer islands is demonstrated in Figure 2B. Surface defects were removed by electropolishing and followed annealing of the copper surface leaded to smooth the surface more. Smoother copper surface provides lower nucleation sites for the graphene and as a result graphene film has less area of few layer. The area of the few layered graphene on the sample with the smooth deposited copper film is comparable with the area on the polished foil and combinations with it (Figure 2C). Despite the minimal roughness of the copper film, it is not possible to completely get rid of the few layer, because it grows at the copper grain edges, unlike the irregularities and impurities in the case of copper foils. It should also be mentioned that most of the impurities on the surface of the copper film are located on the grain boundaries of neighboring crystallites.

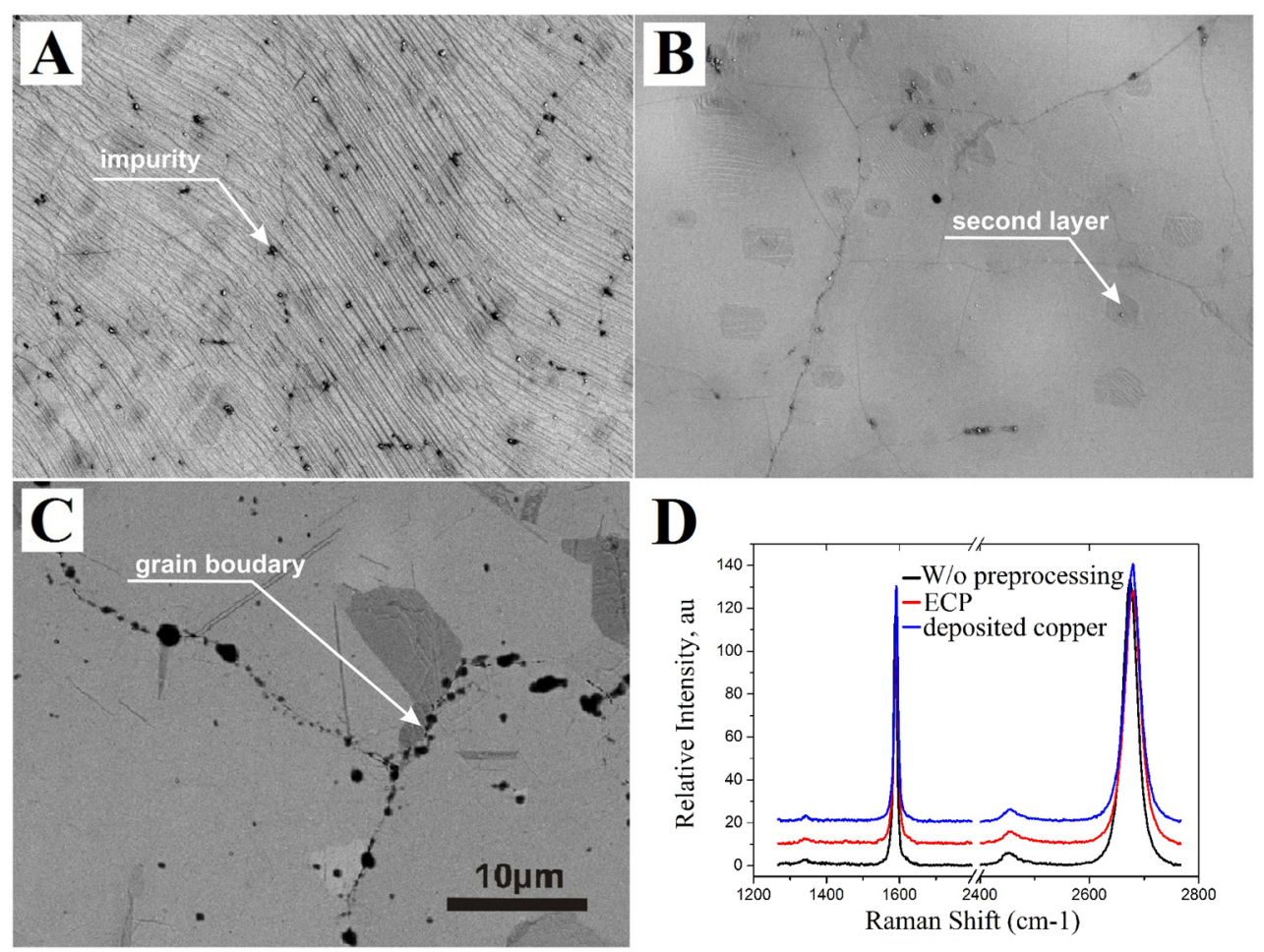

Figure 2. Scanning electron microscopy (SEM) images and Raman spectra of graphene films synthesized on copper foils with different treatment $(\mathbf{A})$ - without pre-treatment, $(\mathbf{B})$ - annealing and polishing) and (C)-deposited copper. The scale bar is the same for all SEM images and presented in (D). 
The Raman spectra in Figure 2D shows that the quality of graphene films is the same for all substrates and does not depend on the surface processing of copper foils, the only difference is the number and average size of islands with few layer graphene film. Measurements of the carrier mobility and sheet resistance, carried out by the four-probe method, have shown values from 2000 to $3000 \mathrm{~cm}^{2} /(\mathrm{V} \cdot \mathrm{s})$ and less than $500 \mathrm{Ohm} / \mathrm{square}$, respectively, indicating the high quality of the synthesized graphene film.

Five regions $\left(100 \times 100 \mu \mathrm{m}^{2}\right)$ of graphene transferred onto silicon oxide were taken from each sample and analyzed using optical microscopy. With a decrease in roughness, the amount of the few layer graphene also decreases. However, with a decrease in roughness below $120 \mathrm{~nm}$, the amount of the few layer graphene remains approximately the same. This can be explained by a decrease in the number of the nucleus (islands) and an increase in the size of islands with a few layer graphene film. For example, for untreated foil $\left(R_{a}=250 \mathrm{~nm}\right)$, more than 1000 islands with an area of $6-9 \mu \mathrm{m}^{2}$ are obtained in the area of $100 \times 100 \mathrm{\mu m}^{2}$, that is, the total area of the few layer graphene film (@) is $6 \div 10 \%$ of the entire surface. After the ECP processing $\left(R_{a}=120 \mathrm{~nm}\right), 150-200$ islands are formed in the same region, but the size of each of them is reduced to $15-20 \mu \mathrm{m}^{2}$ ( $\mathrm{Q}=2 \div 3 \%$ ). In the case of a combination of annealing, chemical etching, and ECP $\left(\mathrm{R}_{\mathrm{a}}=80 \mathrm{~nm}\right)$, less than 50 islands with an area of $50 \mu^{2}$ are formed on the foil, that corresponds to a percentage $\mathrm{Q}=2 \div 3 \%$. On a deposited copper film with a roughness of only $4 \mathrm{~nm}$, the formation of a few layer graphene occurs at the grain boundary of copper crystals, but the size of islands can grow significantly. So only 10-20 islands of the few layer graphene with an area of 50-100 $\mu^{2}$ are observed and their total area takes the same percentage $\varrho=2-3 \%$.

\section{Conclusions}

The chemical vapor deposition technique is an efficient approach for the synthesis of high-quality graphene on copper foil over large areas. The roughness of the copper surface is the key parameter for the fabrication of the most uniform single-layer graphene film. The use of the original commercial copper foil with $\mathrm{R}_{\mathrm{a}}=250 \mathrm{~nm}$ leads to the formation of a few layer graphene film on an area of $9 \%$ of the total surface. Additional processing of catalytic copper foil causes a decrease in the amount of the few layer graphene. The lowest roughness of $80 \mathrm{~nm}$ is achieved using a combination of annealing, etching, and electrochemical polishing, which results in the synthesis of the film with only $2.1 \%$ of the few layer graphene. It is found that the optimal treatment is simply electrochemical polishing, which gives the same amount of a few layer graphene film, but on a copper surface with $\mathrm{R}_{\mathrm{a}}=120 \mathrm{~nm}$. A further decrease in roughness even to $4 \mathrm{~nm}$ does not lead to a decrease in the amount of the few layer graphene.

Acknowledgments: Ivan Kondrashov thanks to RFBR according to the research projects No. 18-2920116_mk for the support of his work. The synthesis of graphene and copper film deposition were done at Prokhorov General Physics Institute and supported by the Russian Science Foundation (Grant No. 18-72-10158). The optical properties of graphene were studied in frames of RFBR project No. 19-02-00-859.

Conflicts of Interest: The authors declare no conflict of interest.

\section{References}

1. Li, X.; Cai, W.; An, J.; Kim, S.; Nah, J.; Yang, D.; Piner, R.; Velamakanni, A.; Jung, I.; Tutuc, E.; et al. Large-area synthesis of highquality and uniform graphene films on copper foils. Science 2009, 324, 1312-1314.

2. McLellan, R.B. The solubility of carbon in solid gold, copper, and silver. Scripta Metallurgica 1969, 3, 389-391.

3. Luo, Z.; Lu, Y.; Singer, D.W.; Berck, M.E.; Somers, L.A.; Goldsmith, B.R.; Johnson, A.C. Effect of substrate roughness and feedstock concentration on growth of wafer-scale graphene at atmospheric pressure. Chem. Mater. 2011, 23, 1441-1447.

4. Sarajlic, O.I.; Mani, R.G. Mesoscale scanning electron and tunneling microscopy study of the surface morphology of thermally annealed copper foils for graphene growth. Chem. Mater. 2013, 25, 1643-1648.

5. Xu, X.; Zhang, Z.; Dong, J.; Yi, D.; Niu, J.; Wu, M.; Lin, L.; Yin, R.; Li, M.; Wang, S.; et al. Ultrafast epitaxial growth of metresized single-crystal graphene on industrial Cu foil. Sci. Bull. 2017, 62, 1074-1080. 
6. Dhingra, S.; Hsu, J.F.; Vlassiouk, I.; D’Urso, B. Chemical vapor deposition of graphene on large-domain ultra-flat copper. Carbon 2014, 69, 188-193.

7. Murdock, A.T.; Van Engers, C.D.; Britton, J.; Babenko, V.; Meysami, S.S.; Bishop, H.; Crossley, A.; Koos, A.A.; Grobert, N. Targeted removal of copper foil surface impurities for improved synthesis of CVD graphene. Carbon 2017, 122, $207-216$.

8. Mun, J.H.; Cho, B.J. Synthesis of monolayer graphene having a negligible amount of wrinkles by stress relaxation. Nano Lett. 2013, 13, 2496-2499.

9. Mohsin, A.; Liu, L.; Liu, P.; Deng, W.; Ivanov, I.N.; Li, G.; Dyck, O.E.; Duscher, G.; Dunlap, J.R.; Gu, G.; et al. Synthesis of millimeter-size hexagon-shaped graphene single crystals on resolidified copper. ACS Nano 2013, 7, 8924-8931.

10. Griep, M.H.; Sandoz-Rosado, E.; Tumlin, T.M.; Wetzel, E. Enhanced graphene mechanical properties through ultrasmooth copper growth substrates. Nano Lett. 2016, 16, 1657-1662.

11. Li, X.; Colombo, L.; Ruoff, R.S. Synthesis of Graphene Films on Copper Foils by Chemical Vapor Deposition. Adv. Mater. 2016, 28, 6247-6252. 